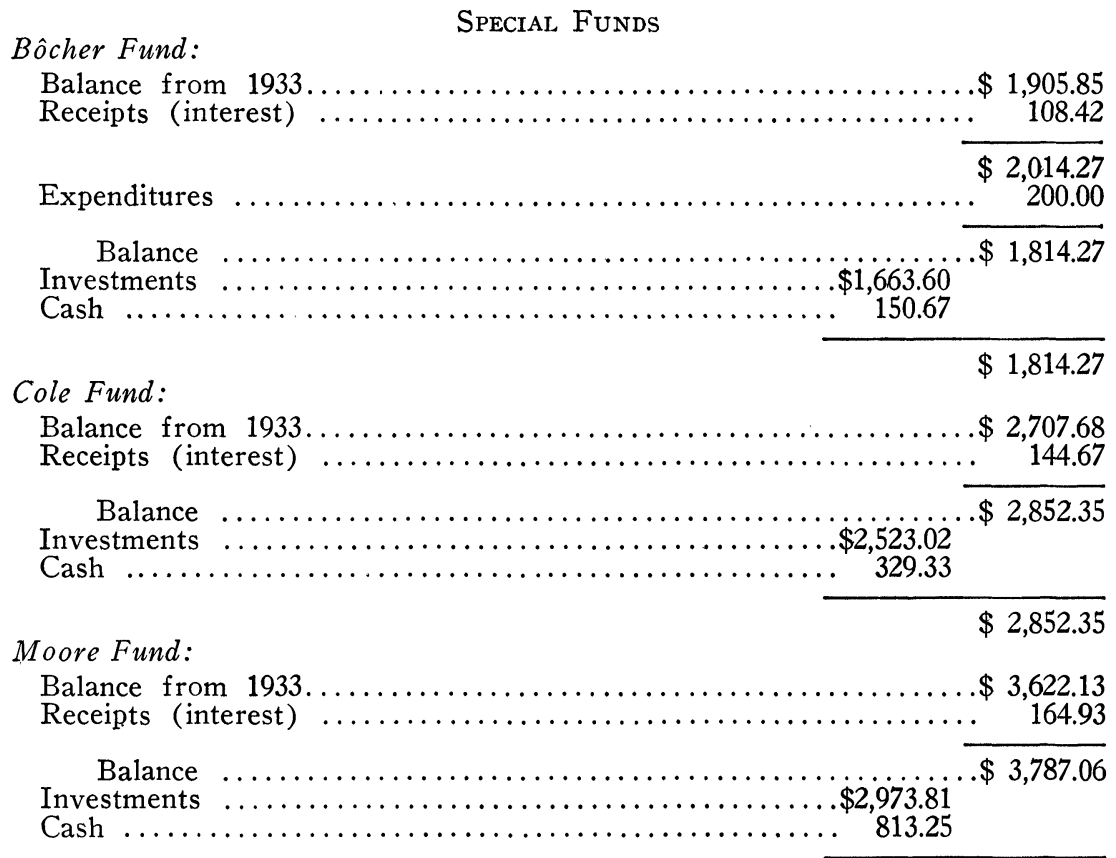
$\$ 6,247.62$.

The amount of reserve necessary to protect the life membership is now

$\begin{array}{ll} & \text { B. P. GiLL } \\ & \text { H. W. Redick } \\ \text { J. J. TANZOLA } & \text { Auditing Committee }\end{array}$

New YoRk, N.Y., December 12, 1934.

\footnotetext{
Receipts :

\section{REPORT OF THE TREASURER FOR THE YEAR 1935} BulletiN Fund

Balance from 1934 (including reprinting fund $\$ 1,576.05$ ) $\ldots \ldots \ldots \ldots \$ 2,920.02$

Appropriation from Dues for current volume.................. $\quad 8,400.00$

Sales, etc. :

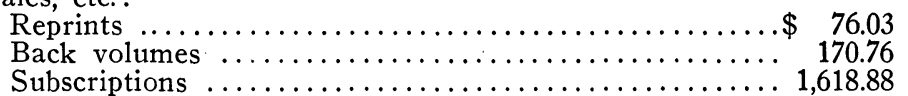

Expenditures :

Printing Bulletin (3 numbers for 1934, 9 numbers for

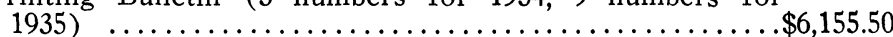

For Treasurer's expenses...................... 344.51

Clerk's annuity ............................... 130.05

Mailing, clerical, insurance, storage, etc............. 2,488.90

Clerical work on 10 -year index...................... 434.33

Balance (including reprinting fund, $\$ 1,312.48$ and reserve for List of Members, $\$ 400) \ldots \ldots \ldots \ldots \ldots \ldots$ 
Receipts :

\section{Transactions Fund}

Balance from 1934 (including reprinting fund \$459.14)

Rockefeller Foundation Subvention $(1935) \ldots \ldots \ldots \ldots \ldots \ldots \ldots \ldots$

Appropriation from Institutional Memberships....................

Appropriation from Individual Contributing Memberships.........

Appropriation from Special Contributions....................

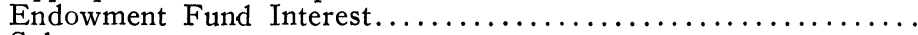

Sales, etc. :

Reprints .............................. 133.01

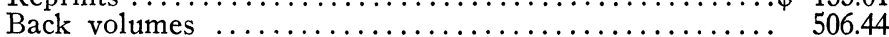

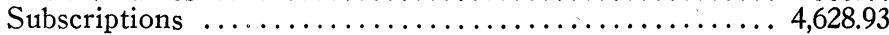

$\$ 2,071.22$

$2,000.00$

550.00

453.50

64.50

$1,500.00$

$5,268.38$

$\$ 11,907.60$

Expenditures:

Printing $(6$ numbers for 1935$) \ldots \ldots \ldots \ldots \ldots \ldots \ldots \ldots \$ 6,648.48$

Mailing, clerical, insurance, storage, etc................ $2,120.67$

$8,769.15$

Balance (including reprinting fund, \$459.14)

$\$ 3,138.45$

Receipts :

\section{Colloguium Fund}

Balance from 1934

Rockefeller Foundation Subvention (1935)

Sales (not including Bliss, Algebraic Functions)*...............

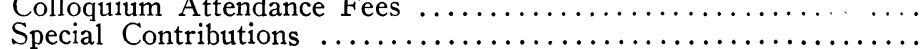

$\$ 3,700.38$

$1,000.00$

$4,486.19$

192.00

100.00

$\$ 9,478.57$

Expenditures :

Printing Paley \& Wiener....................... $\$ 1,480.28$

Customs and duty, importing Jackson and Lefschetz........ 95.84

Shipping, insurance, storage, clerical ................ 912.00

Circulars and advertisements.......................... 70.88

Travelling and honorariums $\ldots \ldots \ldots \ldots \ldots \ldots \ldots \ldots \ldots, 134.46$

$2,693.46$

Balance

$\$ 6,785.11$

\section{General Actrvities}

Balance àt end of 1934 (including $\$ 1,500$ Sinking Fund)

Correction resulting from change in Life Membership Reserve $(\$ 6,588.41-\$ 6,247.62)$

Receipts :

Annual dues $\ldots \ldots \ldots \ldots \ldots \ldots \ldots \ldots \ldots \ldots \ldots \ldots \ldots 11,724.02$

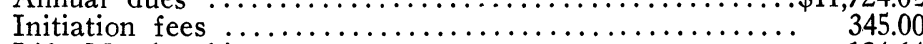

Life Membership .................................. 124.64

Interest

from Endowment Fund........................ 963.03

from General Securities.......................... $\quad 554.76$

from Savings Accounts . . . . . . . . . . . . . . . . . . . . . . . . . 186.95

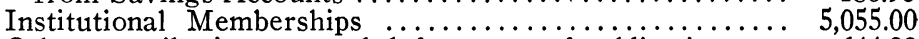

Other contributions toward defrayment of publication cost 644.88

Individual Contributing Memberships................ 453.50

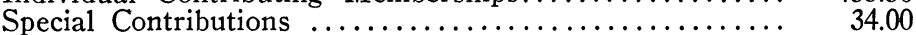

* (Bliss, Algebraic Functions : total receipts, $\$ 366.11$; charge made by Society for shipping, $\$ 53.00$; returned to National Research Council, $\$ 313.11$.) 
Expenditures:

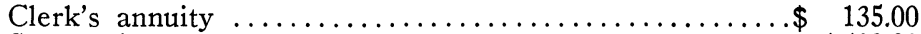

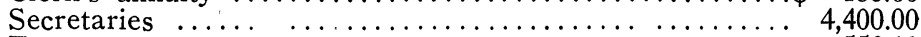

Treasurer ................................... $\quad{ }_{550.00}$

Officers' travelling ............................... 358.51

Membership Committee ........................ 200.00

Euler Subvention ................................. 100.00

Emergency Fund $\ldots \ldots \ldots \ldots \ldots \ldots \ldots \ldots \ldots \ldots \ldots, 451.53$

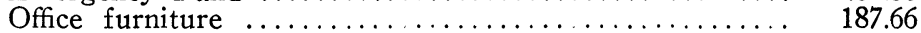

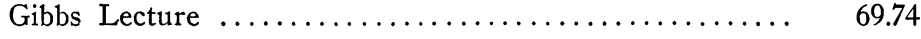

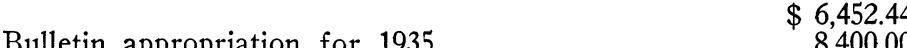

Balance (including $\$ 1,500$ Sinking Fund)......... $\$ 11,337.14$

Receipts :

LIBRARY Fund

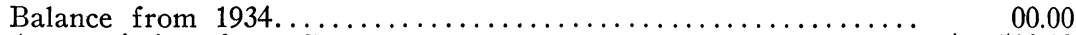

Appropriation from Dues..................................... \$ 500.00

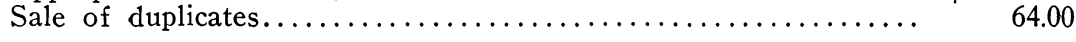

Expenditures: $\$ 564.00$

Binding, clerical, etc............................. 314.32

Balance ........................................... $\overline{\$ 249.68}$

Receipts :

Stabilization Fund

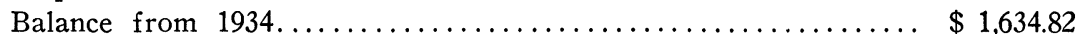

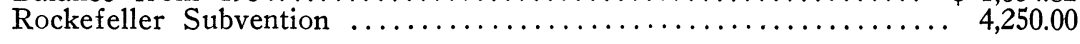

Expenditures: $\quad \frac{\$ 5,884.82}{}$

Salary, travelling expenses, etc.................... $5,823.47$

Balance ..................................... \$ 61.35

American Journal Fund

Rockefeller Foundation Subvention (1935)................. \$1,500.00

Endowment Fund Interest........................... 400.00

Expenditures:

Subvention to American Journal....................... $1,900.00$

Balance ............................................

Life Membership Reserve, $1934 \ldots \ldots \ldots \ldots \ldots \ldots \ldots \ldots \ldots \ldots \ldots \ldots \ldots \ldots$. $\$ 6.247 .62$

Balance, General Fund ..................................... $11,337.14$

Balance, Bulletin Fund........................................ $3,632.40$

Balance, Transactions Fund.................................. $3,138.45$

Balance, Colloquium Fund $\ldots \ldots \ldots \ldots \ldots \ldots \ldots \ldots \ldots \ldots \ldots \ldots \ldots, 6,785.11$

Balance, Stabilization Fund ..................................... ${ }_{61.35}$

Balance, Library Fund ............................... 249.68

$13,673.88$

Cash Balance 
The amount of reserve necessary to protect the life memberships is now $\$ 6,098.80$.

As reported by Mr. Roosevelt, the par value of the securities held by the Society is now $\$ 98,500$. There are now securities of par value $\$ 24,000$ which are defaulting interest payments.

\section{Endowment Fund, Sustaining Memberships, Special Contributions}

\section{Receipts :}

Cash balance, 1934 (Endowment Fund)

Pledges and gifts to Endowment Fund....

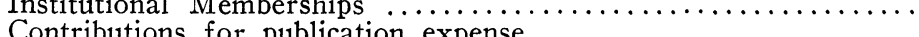

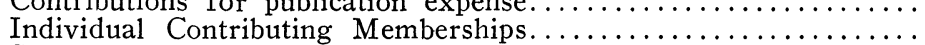

Special Contributions

\section{Expenditures:}

From Institutional Memberships to General Activities....\$5,055.00

From Institutional Memberships to Transactions......... 550.00

From contributions for publication expense to General

Activities ................................. 644.88

From Special Contributions to General Activities.......... 34.00

From Special Contributions to Transactions.............. 64.50

From Special Contributions to Colloquium............... 100.00

From Contributing Memberships to Transactions.......... 453.50

From Contributing Memberships to General Activities... 453.50

Balance

The Endowment Fund has now securities of value $\$ 72,568.26$ (as amortized March, 1934) (including $\$ 100$ Ettlinger Fund). The interest in 1935 (less charges for handling of Fund) was $\$ 2,863.03$.

\section{Bôcher Fund:}

SPECIAL FundS

Balance from 1934:

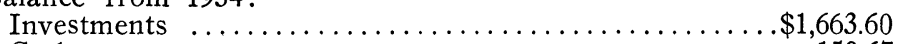

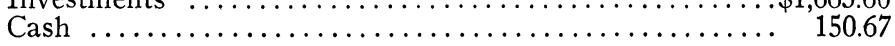

Receipts (interest, 1935) ........................ $\$ 1,814.27$

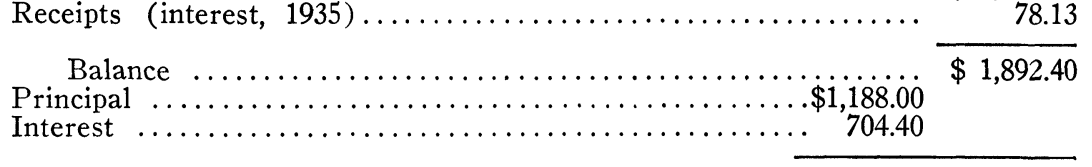

$\$ 1,892.40$

Cole Fund:

Balance from 1934 :

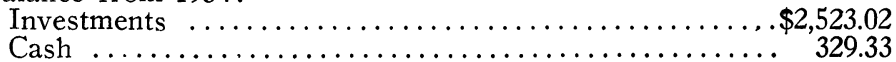

\begin{tabular}{l}
$\$ 2,852.35$ \\
\hline
\end{tabular}

Receipts (interest, 1935).................... ${ }^{2,852.35}$

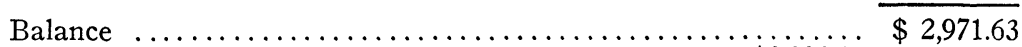

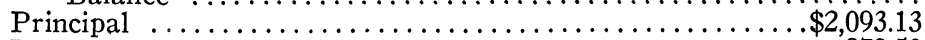

Interest $\ldots \ldots \ldots \ldots \ldots \ldots \ldots \ldots \ldots \ldots \ldots \ldots \ldots \ldots \ldots \ldots \ldots \ldots \ldots \ldots \ldots, 878.50$ 


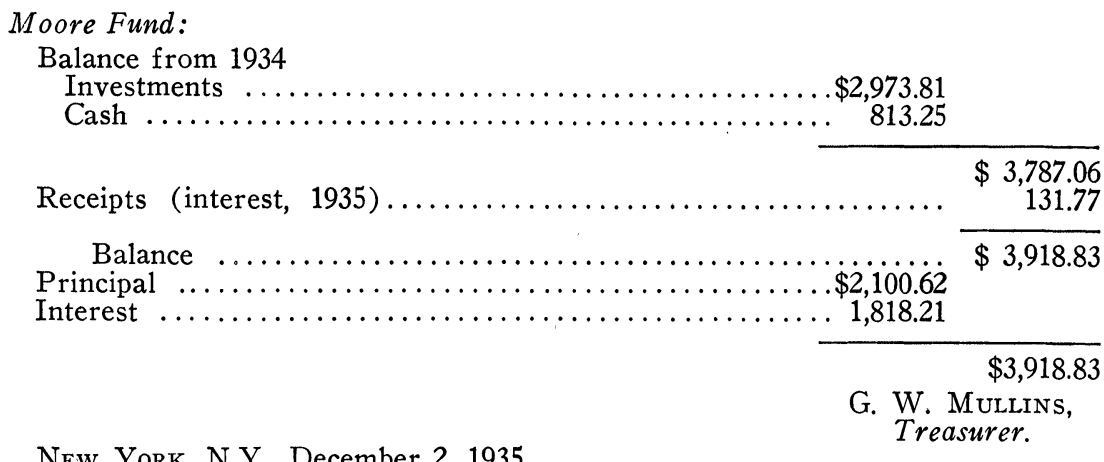

New YoRK, N.Y., December 2, 1935.

\section{REPORT OF THE AUDITING COMMITTEE, 1935}

We, the undersigned Auditing Committee, appointed by the American Mathematical Society, have this day audited the accounts of the Treasurer and in our opinion the following statement is correct:

\section{General Funds}

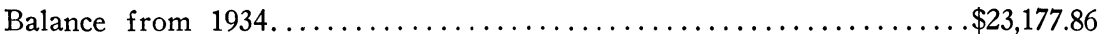

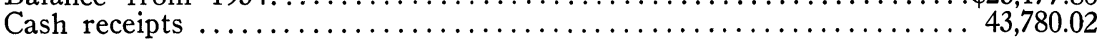

$\$ 66,957.88$

Disbursements

$35,506.13$

Balance, December 2, 1935.

$\$ 31,451.75$

Invested as follows:

Cash in Corn Exchange Bank, exclusive of outstanding checks...... \$6,724.15

Cash in Union Dime Savings Bank ............................ $3,400.72$

Cash in Bank for Savings................................ 2,620.71

Cash in Providence Bank for Savings. . . . . . . . . . . . . . . . . . . . .

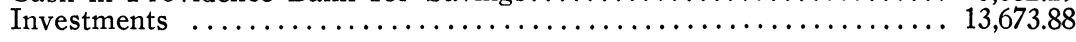

$\$ 31,451.75$

Bôcher Fund:

Special Funds

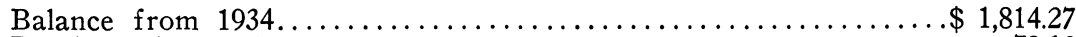

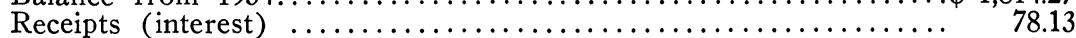

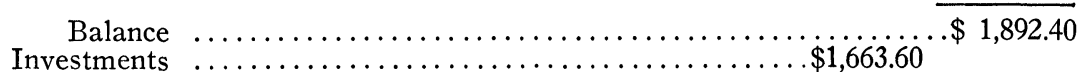

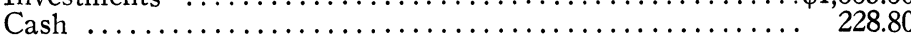

$\$ 1,892.40$

Cole Fund:

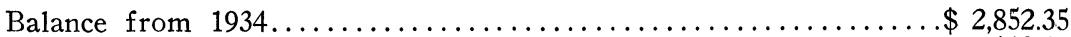

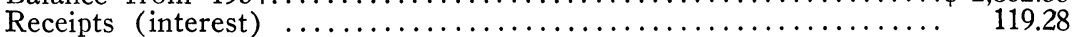

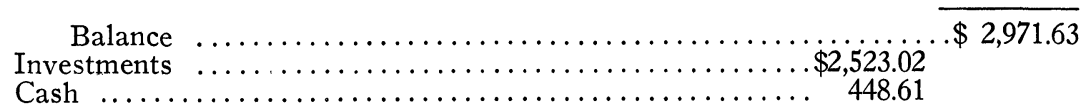

